\title{
Chairmen's Introduction
}

It is my pleasant duty to introduce and to cochair this most interesting symposium on 'Advances in Stroke Prevention'.

Let us first thank the organizers of the Second European Stroke Conference who kindly agreed to host this satellite symposium at a date and time which permitted most participants to attend $\wedge$ and the Sanofi Winthrop Company which generously supported this event and provided the most helpful and competent assistance during its preparation.

The speakers were selected for their well-known competence and expertise in the different domains relevant to stroke prevention. Since 
the Sanofi Symposium at the First European Stroke Conference 2 years ago, there has been an unprecedented flurry of important reports in the first two domains covered by this symposium: pathogenesis and secondary prevention of ischemic stroke. We look forward to hearing updated and critical reviews. Little is known on the social and economical consequences of stroke isthe community, and the data that arc presented in the third part are certainly of utmost importance to the understanding of the true impact of stroke prevention. Each presentation will raise specific Questions, and we encourage the audience to participate aetivelv in the discussions. 